\title{
Valproate Prescribing in Women of Childbearing Age: An Audit of Clinical Practice
}

\author{
Harini Atturu' ${ }^{1}$ and Adedeji Odelola ${ }^{2}$ \\ ${ }^{1}$ Pennine Care NHS Foundation Trust, Royal Oldham Hospital, Oldham, Lancashire OL1 2JH, UK \\ ${ }^{2}$ Pennine Care NHS Foundation Trust, Birch Hill Hospital, Rochdale OL12 9QB, UK
}

Correspondence should be addressed to Adedeji Odelola; deji.odelola@nhs.net

Received 16 June 2014; Revised 13 January 2015; Accepted 22 January 2015

Academic Editor: Holly A. Garriock

Copyright ( $) 2015$ H. Atturu and A. Odelola. This is an open access article distributed under the Creative Commons Attribution License, which permits unrestricted use, distribution, and reproduction in any medium, provided the original work is properly cited.

\begin{abstract}
Background. Evidence is accruing regarding the risks of valproate exposure in women of childbearing age. Recommendations have recently been made for a higher standard of prenatal counselling and prescribing practice in respect of valproate use in this patient group. Aim and Method. A reaudit was carried out to review the standard of clinical discussion around teratogenic risk and pregnancy planning offered to women of child-bearing age prescribed valproate. Case notes and prescription charts of women 45 years old or less were examined and compared with the results of a previous audit in 2005. Results. The use of valproate was increased overall by $64 \%$ and there was an $18 \%$ increase in off-label valproate use. The rate of clinical discussion carried out during commencement declined from $70 \%$ to $35 \%$ and at annual review from $50 \%$ to $22 \%$. There was less clinical discussion in outpatients and in older patients. More than $40 \%$ of doctors surveyed were not confident about giving information to women. Clinical Implication. There is a need for a multidisciplinary approach and action at Healthcare Trust level, to increase awareness and reduce risks associated with valproate prescribing in childbearing women.
\end{abstract}

\section{Introduction}

The risks in women of childbearing age associated with prenatal valproate exposure are becoming established [1], and updated recommendations are being made nationally and internationally for restrictions to valproate use and enhanced preconceptual counselling in this patient group [2,3]. Recent research has shown that in utero exposure to sodium valproate is associated with a range of neuropsychological, language, and developmental difficulties [4-9] and a higher incidence of autistic spectrum disorder compared to controls [10].

Valproate is now considered to be the most teratogenic of all the antiepileptics [11, 12]. Exposure to valproate doses greater than or equal to $1000 \mathrm{mgs}$ is generally associated with a higher range of teratogenic risk (21.9\%), compared to $2.5 \%$ with lower doses, and a fourfold increase in the rate of major anomalies is reported with antiepileptic polytherapy $[1,13]$. Valproate is associated with a teratogenic risk of $6.3 \%$ compared to other medicines like carbamazepine (2.4\%) and lamotrigine $(2.7 \%)$. Lithium is associated specifically with a $0.1 \%$ risk of Ebstein's cardiacanomaly [14]. The least risk is attributed to antipsychotics $[12,15]$.

It is now not recommended that valproate should be prescribed routinely for women with child-bearing potential. If valproate use is inevitable, then recommendations are for a clinical discussion involving teratogenic risk, pregnancy planning, and contraception advice $[1-3,16]$.

In July 2005, a local audit showed inadequate clinical discussion associated with valproate prescribing, and specially designed "yellow monitoring forms" were developed to facilitate clinical discussion and documentation during initiation and follow-up of women prescribed valproate.

The current reaudit is to examine the extent to which clinical discussions regarding the teratogenic potential of valproate, contraceptive advice, and pregnancy planning are offered to women of child bearing age during commencement of valproate treatment and at review. 
TABLE 1: Rates for clinical discussion in 2005 and 2012 audits.

\begin{tabular}{|c|c|c|c|c|}
\hline Audit standard & Commencement $2005(\%)$ & Commencement $2012(\%)$ & Review $2005(\%)$ & Review $2012(\%)$ \\
\hline Any clinical discussion documented & $70 \%$ & $35 \%$ & $50 \%$ & $22 \%$ \\
\hline Discussion of teratogenicity & $70 \%$ & $35 \%$ & $50 \%$ & $22 \%$ \\
\hline Discussion about contraception & $70 \%$ & $35 \%$ & $50 \%$ & $22 \%$ \\
\hline Collaborative pregnancy planning discussed & $70 \%$ & $35 \%$ & $50 \%$ & $22 \%$ \\
\hline Last menstrual period documented & $70 \%$ & $30 \%$ & - & $13 \%$ \\
\hline Communication with GP & $70 \%$ & $25 \%$ & $50 \%$ & $13 \%$ \\
\hline
\end{tabular}

\section{Method}

The current reaudit involved women between the ages of 18 to 45 years under the care of the Rochdale General Adult Psychiatric service from August 2005 till October 2012, receiving valproate preparations for psychiatric illness. Women taking valproate primarily for epilepsy were excluded as they are generally managed by primary care or epilepsy services. Using search terms "Valproate," "Depakote," or "Epilim" patients were identified from the department's electronic patient administration database.

Audit standards were set at a departmental meeting and were derived from a previous departmental audit done in 2005 and reflected guidance by NICE [16]. Agreed standards were the following. (1) For women of childbearing age, there should be evidence of a clinical discussion at initiation of valproate prescribing. (2) There should be a further discussion at subsequent clinical review for women prescribed valproate. (3) The clinical discussion should include the provision of contraceptive advice, discussion of the need for pregnancies to be planned collaboratively with the prescriber, a discussion of the risk of teratogenicity, and a record of the patient's last menstrual period (LMP). (4) Details of the clinical discussion should be communicated to the patient's General Practitioner. (5) Yellow monitoring forms developed after the previous audit (2005), specifically for the recording of information provided to patients, should be completed for each patient and filed in their case notes.

Consecutive patients who were under the care of either of two community sector teams were included in the study. One of the study authors (Harini Atturu) reviewed patient case notes and searched for the presence of the "yellow monitoring forms." In addition the same author examined handwritten case note entries and written correspondence for any other evidence of clinical discussion, contraceptive advice, or pregnancy planning contained within the records. Case notes were searched for evidence of adverse outcomes occurring in patients who received valproate during pregnancy and these were recorded as "near misses." Results were compared with those of the previous audit using basic statistics specifically prevalence rates expressed as percentages.

A separate survey was carried out prior to discussion of the audit findings, which included doctors within the department attending an educational meeting in January 2013. All doctors of various grades attending the meeting were invited to take part in the survey. Survey questions were agreed by consensus by both authors (Harini Atturu and Adedeji Odelola), based on the results of the previous
TABLE 2: Use of valproate for different psychiatric disorders.

\begin{tabular}{lcc}
\hline Diagnosis & $\begin{array}{c}\text { July 2005 } \\
(n=14)\end{array}$ & $\begin{array}{c}\text { October 2012 } \\
(n=23)\end{array}$ \\
\hline Bipolar disorder & $78.7 \%$ & $60.8 \%$ \\
Schizoaffective disorder & $7.1 \%$ & $17.4 \%$ \\
Paranoid schizophrenia & $7.1 \%$ & $8.7 \%$ \\
Psychotic depression & $7.1 \%$ & $4.3 \%$ \\
Personality disorder & $0 \%$ & $8.7 \%$ \\
\hline
\end{tabular}

audit (2005), and sought to uncover reasons for the poor practice identified in that audit. Questions were kept openended as much as possible, inviting a narrative response, and covered choice of medication, barriers to clinical discussion with women of childbearing age, and the scope of such discussions.

\section{Results}

In the 2012 audit, a total of 94 patients were identified from the database. Out of these 46 were male and 48 were female. 26 patients were women up to 45 years in age. Three women had a diagnosis of epilepsy and were excluded. 23 cases met the study criteria. The initial audit carried out in 2005 involved a sample of 14 women who received valproate.

The results in respect of clinical discussions carried out in 2005 are shown in Table 1 and are shown alongside findings in the current (2012) audit.

\section{Diagnosis}

Compared with the previous (2005) study, the present study found an increase in the use of valproate for psychiatric disorders other than bipolar disorders. The use of valproate for schizoaffective disorder and paranoid schizophrenia was increased. There were no cases of valproate use for personality disorder in 2005 but $8.7 \%$ of the 2012 sample received valproate for a personality disorder. The routine use of valproate for bipolar disorder and for depression with psychosis was found to be reduced (Table 2).

\section{Age}

The use of valproate in women less than or equal to 25 years of age had reduced from $50 \%$ to $4.3 \%$ when compared with the 2005 audit. The use of valproate had remained essentially 
TABLE 3: Trends in the usage of valproate in different settings.

\begin{tabular}{lccc}
\hline Different settings & $\begin{array}{c}\text { \% of patients on valproate } \\
\text { in 2005 }(n=14)\end{array}$ & $\begin{array}{c}\text { \% of patients on valproate } \\
\text { in 2012 }(n=23)\end{array}$ & $\begin{array}{c}\text { Total change in usage of valproate } \\
\text { between } 2005 \text { and } 2012\end{array}$ \\
\hline Inpatient & $35 \%$ & $43.5 \%$ & $8.5 \%$ increase \\
Outpatient & $50 \%$ & $43.5 \%$ & $6.5 \%$ decrease \\
Other teams & $14.3 \%$ & $13 \%$ & $1.3 \%$ decrease \\
\hline
\end{tabular}

TABLE 4: Clinical discussions in respect of valproate prescribing during 2012 audit.

\begin{tabular}{|c|c|c|c|c|c|c|}
\hline Age group & Number of pts & Teratogenicity & Contraceptive advice & Pregnancy planning & LMP & GP advised \\
\hline$<25 y$ & 1 & $100 \%$ & $100 \%$ & $100 \%$ & $0 \%$ & $100 \%$ \\
\hline $26-35 y$ & 5 & $40 \%$ & $40 \%$ & $40 \%$ & $40 \%$ & $40 \%$ \\
\hline $36-45 y$ & 14 & $29 \%$ & $29 \%$ & $29 \%$ & $29 \%$ & $14 \%$ \\
\hline Commencement (all ages) & 20 & $35 \%$ & $35 \%$ & $35 \%$ & $30 \%$ & $25 \%$ \\
\hline Review (all ages) & 23 & $22 \%$ & $22 \%$ & $22 \%$ & $13 \%$ & $13 \%$ \\
\hline
\end{tabular}

the same in the age group 26-35 years. However, in age group $36-45$ years, the use of valproate had increased from $21.4 \%$ to $69.6 \%$.

\section{Use of Valproate in Inpatient and Outpatient Settings}

There was an increased trend in the prescribing of valproate by $8.5 \%$ in inpatients and a decrease by $6.5 \%$ in outpatients compared with the previous audit. Additionally, a small group of patients commenced valproate with the Home Treatment Team, or in an external setting prior to transfer to our hospital. In these patients there was a very slight decrease in levels of valproate prescribing of $1.3 \%$ compared with the previous audit (Table 3 ).

\section{Clinical Discussions in respect of Valproate Prescribing}

The current study found a decline in the rate of clinical discussion carried out during commencement from $70 \%$ to $35 \%$ and at annual review from $50 \%$ to $22 \%$ compared with the 2005 audit. The 2012 audit found that, during initiation of valproate for inpatients, clinical discussions were carried out and documented $60 \%$ of the time. In the outpatient setting, clinical discussion was carried out only $10 \%$ of the time. There was a trend observed for clinical discussions to be carried out with less frequency in older patient groups (Table 4).

\section{Documentation}

The previous (2005) audit recommended that "yellow monitoring forms" were completed and filed in the case-notes for all women of child-bearing age commencing valproate. However, during clinic reviews in the 2012 audit, the yellow form was available in only $4.3 \%$ of cases. In patients without "Yellow Forms" there was nevertheless, evidence at review, of explaining teratogenic risks, giving of the contraceptive and collaborative pregnancy planning advice in $22 \%$ of cases and evidence of recording of last menstrual periods and communication of the clinical discussion to general practitioners in $13 \%$ (Table 4 ).

"Near Misses". Two women were identified during the 2012 audit who were classed as "near misses." These were women in the study sample who became pregnant during the study period. The first patient was commenced on valproate treatment without a clinical discussion. She however did have a clinical discussion at outpatient review, was appropriately counselled, and chose to discontinue valproate prior to becoming pregnant. A second patient had a clinical discussion at commencement of valproate treatment but defaulted from follow-up and hence did not have an outpatient review. She became pregnant and delivered a preterm baby with mild intracranial haemorrhage.

\section{Survey}

Following the reaudit, we carried out a survey among doctors locally, to uncover the reasons for the poor performance in respect of valproate prescribing advice. 17 doctors participated out of whom $23.5 \%$ were consultants, $29 \%$ were speciality trainee/speciality doctors, $23.5 \%$ were core trainees, $12 \%$ were foundation trainees, and $12 \%$ did not specify their grade.

94\% of doctors in the sample stated that they would not consider valproate as first choice while treating women of child bearing age. $6 \%$ of doctors did not know what information ought to be given to women, and $41 \%$ admitted that they only gave partial information. $12 \%$ reported using yellow forms and only 35\% said that they communicated their clinical discussion by letter to general practitioners in addition to documentation in case notes.

In carrying out clinical discussions, doctors identified a few barriers. These included time pressure (12\%); patient factors like patient capacity and communication difficulties (12\%), lack of knowledge (6\%) and nonspecific factors (6\%). More than $40 \%$ of doctors felt that they had low (12\%) or 
moderate (30\%) levels of confidence about their knowledge in respect of giving valproate prescribing advice.

\section{Discussion}

There is limited information in the literature regarding prescribing patterns of antiepileptic drugs in the United Kingdom. Specifically in patients with bipolar disorder, however, the trend has been for a steady increase in valproate prescribing since 1995, with increased psychotropic drug coprescribing $[17,18]$. In the United States, valproate prescription has remained the same amongst women of childbearing age, despite the teratogenic risks [19]. Our study results were similar to those of other studies in our finding of an overall rate of usage of valproate increased by $64 \%$ compared with the previous (2005) audit. An increased rate of usage from $24 \%$ to $62 \%$ was reported in a study in Newcastle, United Kingdom [20].

In the current study there was an increase in the prescription of sodium valproate by $8.5 \%$ when commenced in inpatients and a $6.5 \%$ decrease in outpatients-perhaps reflecting the requirement for effective medicines to manage severely ill patients in the inpatient setting. The trend appears to be shifting from the use of valproate predominantly for bipolar disorder to the use in several other psychiatric disorders like schizoaffective disorder, paranoid schizophrenia, and personality disorder showing flexible use for different conditions within psychiatry. This trend for off-license prescribing has been shown in other studies [21].

Valproate use was found to be reduced in our study, from $50 \%$ to $4.3 \%$ in women in younger age groups $(\leq 25$ years), compared with an increased use from $21.4 \%$ to $69.6 \%$ in women in the $36-45$ years age group who might have completed their families. This trend is similar to that reported in other studies. An Australian study found that neurologists involved in the care of women with epilepsy had changed their pattern of prescribing based on knowledge of the teratogenic risks with valproate [22], prescribing less frequently and in lower dosage. A Primary Care study looking into trends of prescribing of valproate in adolescent females found increased prescribing of lamotrigine but decreased use of carbamazepine and a $3.1 \%$ reduction in the use of valproate [23].

It is not clear whether or not the results of our study in respect of younger women are a true reflection of improved prescribing practice or have arisen because our younger population is served by an Early Intervention team. It may be that this question would be addressed by doing similar valproate prescribing audits of Early Intervention teams which are more involved in the care of transition populations.

$50 \%$ or more of pregnancies occur unplanned; hence it is generally recommended that contraception issues should be discussed regardless of whether or not women are planning a pregnancy [12]. The literature shows that women with bipolar disorder encounter problems engaging with health care professionals regarding their pregnancy planning. A survey among women with bipolar disorder showed that a preconceptual consultation, involving an explanation of the risks and benefits of maintenance or discontinuation of treatment in pregnancy, had an influence on patient's decisions. $37 \%$ of women chose not to pursue pregnancy while $63 \%$ decided to conceive following preconceptual consultation [24].

A 20-month Quality Improvement Programme in the South London and Maudsley NHS Foundation Trust demonstrated a significant improvement in information provision following the use of a multidisciplinary approach with both doctors and pharmacists involved in counselling women on the risks of medication in pregnancy. The study also found that ongoing identification of women prescribed valproate by the pharmacist and subsequent reminders to prescribers to counsel and advise women were effective [25]. We found a higher rate of discussion carried out during inpatient commencement (60\%), compared to $10 \%$ in the outpatient setting. This could be attributed to the availability of a pharmacist on our wards and may highlight the need for employing a multidisciplinary approach in the outpatient setting as well.

The current reaudit showed an overall decline in rate of clinical discussions carried out during commencement from $70 \%$ to $35 \%$ and at review from $50 \%$ to $22 \%$. Similar low rates of clinical discussion have been found in other published audits of mood stabiliser prescribing. An audit carried out in Bradford District Care Trust showed that in women of child bearing age using various mood stabilisers contraception advice was given, and risk of pregnancy was verbally communicated in 35\% of cases and contraception advice was documented in only $24 \%$ of cases [26]. There were similarly low rates obtained in a survey of three Greater Manchester Teaching Hospitals [27] and a case-note review carried out in Kent [28].

The Bradford group found one of the main reasons for this low rate of clinical discussion was poor documentation [26]. Our survey of doctors found that a proportion of doctors claimed that they tended to hold discussions but did not usually indicate this by clear documentation in case notes. Our survey results also showed that more than $40 \%$ of doctors felt low to moderate levels of confidence about their knowledge in this area, indicating a training need. Other survey results suggest that, in addition to a lack of knowledge and confidence, factors specific to the doctorpatient interaction and a particular difficulty imparting the requisite information to women may be involved in the low rate of clinical discussion obtained in our audit.

The yellow monitoring forms introduced at the time of the initial audit in 2005 would have facilitated clinical discussion by serving as a template offering prompts for the various areas needing to be discussed. The reasons for the low rate of usage in our study population of the yellow monitoring forms are unclear. It may be that there is a role for local pharmacists in respect of increasing awareness and accessibility of the yellow monitoring forms and identifying women falling within the at-risk group [25].

In the current study there were two cases which were identified as being "near misses" in whom adverse outcomes due to valproate use during pregnancy were narrowly averted. One woman did not have a clinical discussion at 
commencement of valproate treatment but was identified at outpatient clinic review, discontinued valproate, and became pregnant. The consequences for the foetus may have been severe had there not been a clinical discussion at review. A second patient who received adequate information provided at commencement of valproate treatment, became pregnant having dropped out of services, and delivered a preterm baby with mild intracranial haemorrhage. This outcome may have been prevented had it been possible to have an outpatient review but in any case highlights the need for effective liaison and communication with general practitioners in respect of women prescribed valproate preparations. A similar study, reviewing mood stabiliser prescribing practice in Kent [28], found that 14 women (10\%) became pregnant while taking lithium, carbamazepine, or valproate and 8 women had a complication of pregnancy.

One limitation of the current reaudit is that it only involved patients under the care of Adult Community Mental Health Teams. The current study did however involve all women prescribed valproate during the study period which extended from the time of the conclusion of the previous audit in 2005, to the current time (2012). There would no doubt be benefits from future studies involving a wider range of community services such as Early Intervention teams, Adult Intellectual Disability teams, and Child and Adolescent services. In addition, although the current reaudit involved patients prescribed valproate since 2005 , the reaudit was done in 2012-7 years later. It is likely that an earlier reaudit would have helped to maintain awareness of the need for appropriate clinical discussion.

\section{Conclusion}

In the current audit, the level of clinical discussion with women prescribed valproate was found to be less than adequate. There is a need for strategic planning by Healthcare Trusts in accordance with national guidance in respect of valproate prescribing. We recommend a multidisciplinary approach involving pharmacists, increasing awareness by means of induction programmes for doctors, and the use of specific aids for the provision of information such as alert labels, patient leaflets, and the monitoring forms developed after the previous valproate audit.

\section{Conflict of Interests}

The authors declare that there is no conflict of interests regarding the publication of this paper.

\section{References}

[1] T. Tomson, D. Battino, E. Bonizzoni et al., "Dose-dependent risk of malformations with antiepileptic drugs: an analysis of data from the EURAP epilepsy and pregnancy registry," The Lancet Neurology, vol. 10, no. 7, pp. 609-617, 2011.

[2] European Medicines Agency, "PRAC recommends strengthening the restrictions on the use of valproate in women and girls. Review 612389," London, UK, European Medicines Agency, 2014.
[3] National Institute for Health and Care Excellence (NICE), "Antenatal and postnatal mental health: clinical management and service guidance," in Clinical Guideline 192, National Institute for Health and Care Excellence, 2014.

[4] S. J. Moore, P. Turnpenny, A. Quinn et al., "A clinical study of 57 children with fetal anticonvulsant syndromes," Journal of Medical Genetics, vol. 37, no. 7, pp. 489-497, 2000.

[5] N. Adab, U. Kini, J. Vinten et al., "The longer term outcome of children born to mothers with epilepsy," Journal of Neurology, Neurosurgery and Psychiatry, vol. 75, no. 11, pp. 1575-1583, 2004.

[6] K. J. Meador, G. A. Baker, N. Browning et al., "Cognitive function at 3 years of age after fetal exposure to antiepileptic drugs," The New England Journal of Medicine, vol. 360, no. 16, pp. 1597-1605, 2009.

[7] E. Gaily, E. Kantola-Sorsa, V. Hiilesmaa et al., "Normal intelligence in children with prenatal exposure to carbamazepine," Neurology, vol. 62, no. 1, pp. 28-32, 2004.

[8] J. Vinten, N. Adab, U. Kini, J. Gorry, J. Gregg, and G. A. Baker, "Neuropsychological effects of exposure to anticonvulsant medication in utero," Neurology, vol. 64, no. 6, pp. 949-954, 2005.

[9] E. Kantola-Sorsa, E. Gaily, M. Isoaho, and M. Korkman, "Neuropsychological outcomes in children of mothers with epilepsy," Journal of the International Neuropsychological Society, vol. 13, no. 4, pp. 642-652, 2007.

[10] R. L. Bromley, G. Mawer, J. Clayton-Smith, and G. A. Baker, "Autism spectrum disorders following in utero exposure to antiepileptic drugs," Neurology, vol. 71, no. 23, pp. 1923-1924, 2008.

[11] A. Ornoy, "Neuroteratogens in man: an overview with special emphasis on the teratogenicity of antiepileptic drugs in pregnancy," Reproductive Toxicology, vol. 22, no. 2, pp. 214-226, 2006.

[12] G. M. Goodwin, "Evidence-based guidelines for treating bipolar disorder: revised second edition-recommendations from the British association for psychopharmacology," Journal of Psychopharmacology, vol. 23, no. 4, pp. 346-388, 2009.

[13] O. Diav-Citrin, S. Shechtman, B. Bar-Oz, D. Cantrell, J. Arnon, and A. Ornoy, "Pregnancy outcome after In Utero exposure to valproate: evidence of dose relationship in teratogenic effect," CNS Drugs, vol. 22, no. 4, pp. 325-334, 2008.

[14] R. F. McKnight, M. Adida, K. Budge, S. Stockton, G. M. Goodwin, and J. R. Geddes, "Lithium toxicity profile: a systematic review and meta-analysis," The Lancet, vol. 379, no. 9817, pp. 721728, 2012.

[15] Epilepsy Guidance Group, Primary Care Guidance for the Management of Women taking Antiepileptic Drugs, Epilepsy Guidance Group, 2011.

[16] National Institute for Health and Clinical Excellence (NICE), Bipolar Disorder: The Management of Bipolar Disorder in Adults, Children and Adolescents, in Primary and Secondary Care, NICE Clinical Guidelines, no. 38, NICE, 2006.

[17] J. Hayes, P. Prah, I. Nazareth et al., "Prescribing trends in bipolar disorder: cohort study in the United Kingdom THIN primary care database 1995-2009," PLoS ONE, vol. 6, no. 12, Article ID e28725, 2011.

[18] National Institute for Health and Clinical Excellence (NICE), NICE Implementation Uptake Report: Bipolar Disorder Clinical Guideline 38, NICE, 2009.

[19] D. A. Adedinsewo, D. J. Thurman, Y. H. Luo, R. S. Williamson, O. A. Odewole, and G. P. Oakley Jr., "Valproate prescriptions for nonepilepsy disorders in reproductive-age women," Birth 
Defects Research, Part A-Clinical and Molecular Teratology, vol. 97, no. 6, pp. 403-408, 2013.

[20] A. J. Lloyd, C. L. Harrison, I. N. Ferrier, and A. H. Young, "The pharmacological treatment of bipolar affective disorder: practice is improving but could still be better," Journal of Psychopharmacology, vol. 17, no. 2, pp. 230-233, 2003.

[21] J. Langan, A. Perry, and M. Oto, "Teratogenic risk and contraceptive counselling in psychiatric practice: analysis of anticonvulsant therapy," BMC Psychiatry, vol. 13, article 234, 2013.

[22] F. J. E. Vajda, S. Hollingworth, J. Graham et al., "Changing patterns of antiepileptic drug use in pregnant Australian women," Acta Neurologica Scandinavica, vol. 121, no. 2, pp. 89-93, 2010.

[23] R. Ackers, F. M. C. Besag, A. Wade, M. L. Murray, and I. C. $\mathrm{K}$. Wong, "Changing trends in antiepileptic drug prescribing in girls of child-bearing potential," Archives of Disease in Childhood, vol. 94, no. 6, pp. 443-447, 2009.

[24] A. C. Viguera, L. S. Cohen, S. Bouffard, T. H. Whitfield, and R. J. Baldessarini, "Reproductive decisions by women with bipolar disorder after prepregnancy psychiatric consultation," The American Journal of Psychiatry, vol. 159, no. 12, pp. 21022104, 2002.

[25] S. Mace and D. Taylor, "Improving adherence to NICE guidance for bipolar illness: valproate use in women of childbearing potential," The Psychiatric Bulletin, vol. 35, no. 2, pp. 63-67, 2011.

[26] M. Mothi, C. Chambers, S. Shora, and A. Jabeen, "2305-Audit on effects of mood stabilisers on women's health-informed decision making," European Psychiatry, vol. 28, supplement 1, p. 1, 2013.

[27] A. Wieck, S. Rao, K. Sein, and P. M. Haddad, "A survey of antiepileptic prescribing to women of childbearing potential in psychiatry," Archives of Women's Mental Health, vol. 10, no. 2, pp. 83-85, 2007.

[28] L. James, T. R. E. Barnes, P. Lelliott, D. Taylor, and C. Paton, "Informing patients of the teratogenic potential of mood stabilizing drugs: a case note review of the practice of psychiatrists," Journal of Psychopharmacology, vol. 21, no. 8, pp. 815-819, 2007. 


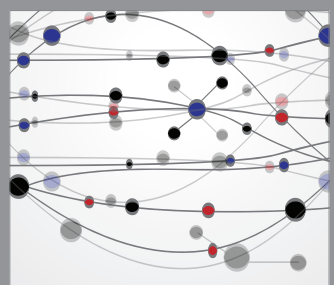

The Scientific World Journal
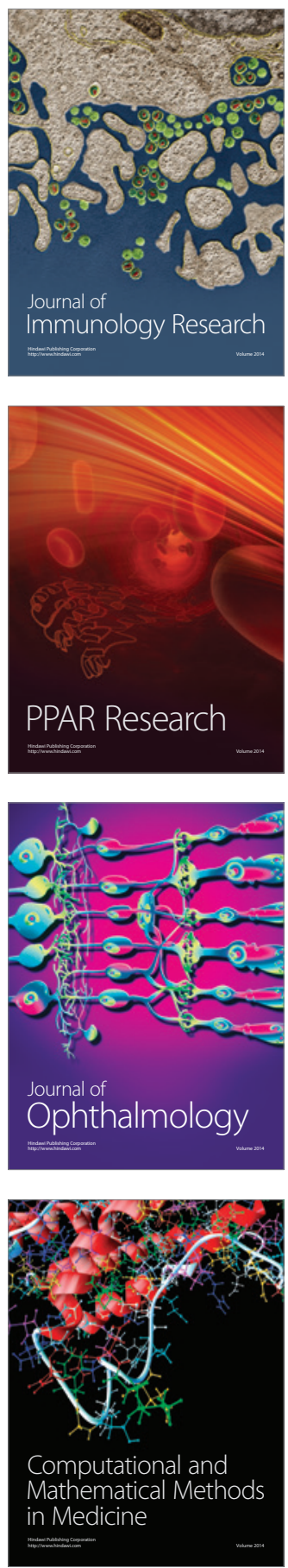

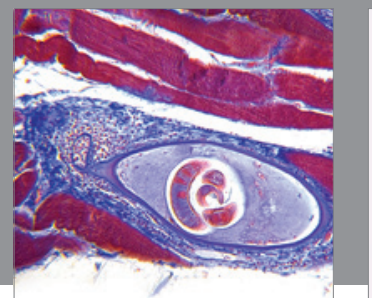

Gastroenterology

Research and Practice
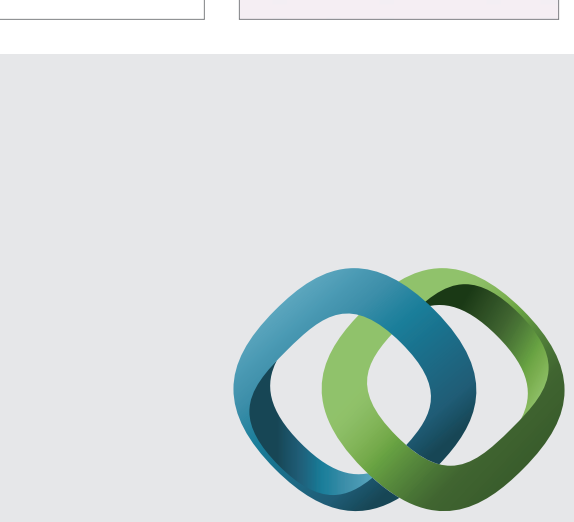

\section{Hindawi}

Submit your manuscripts at

http://www.hindawi.com
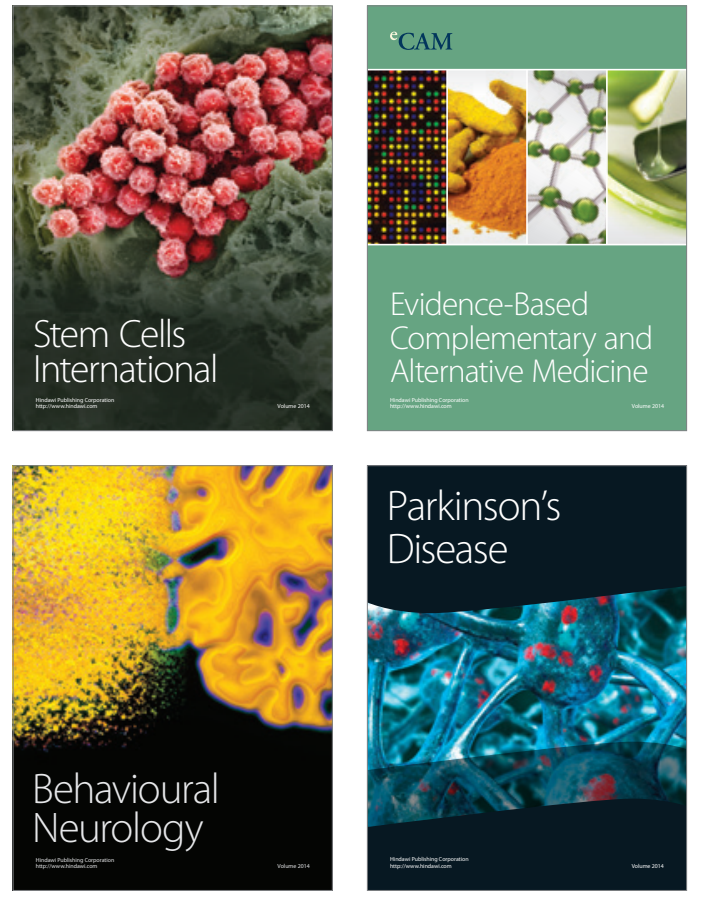
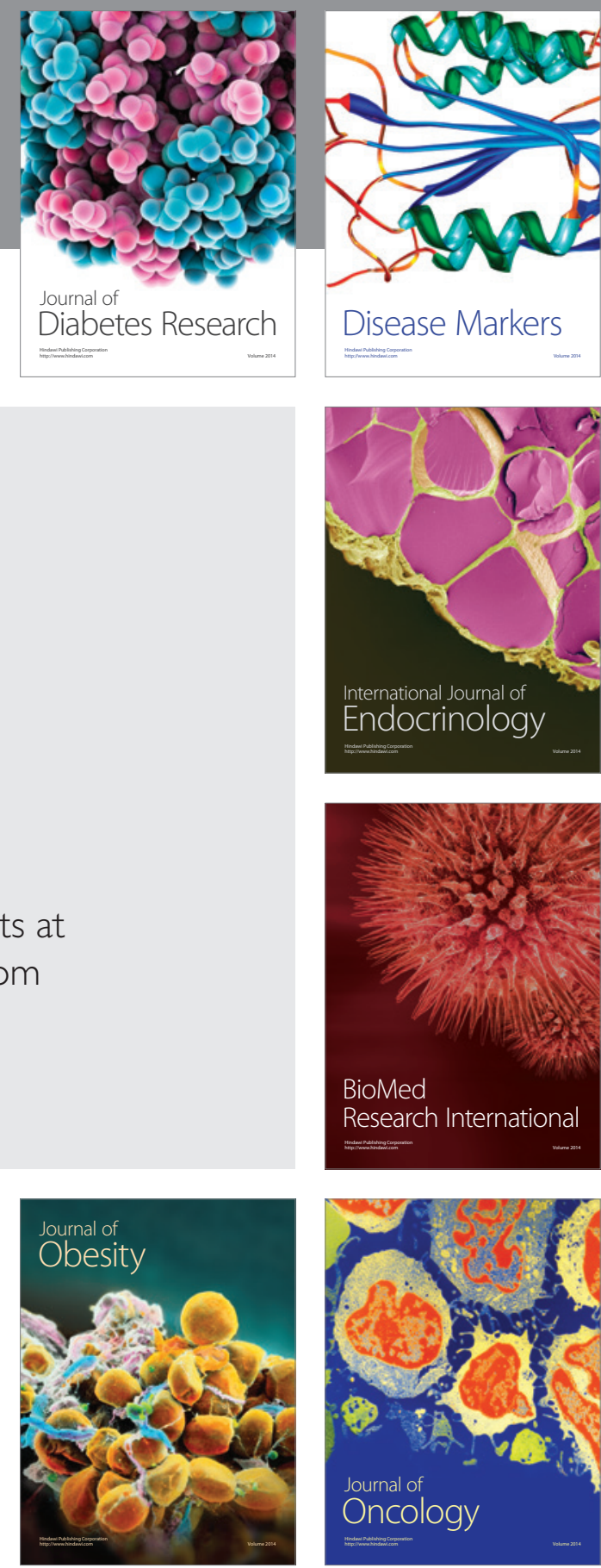

Disease Markers
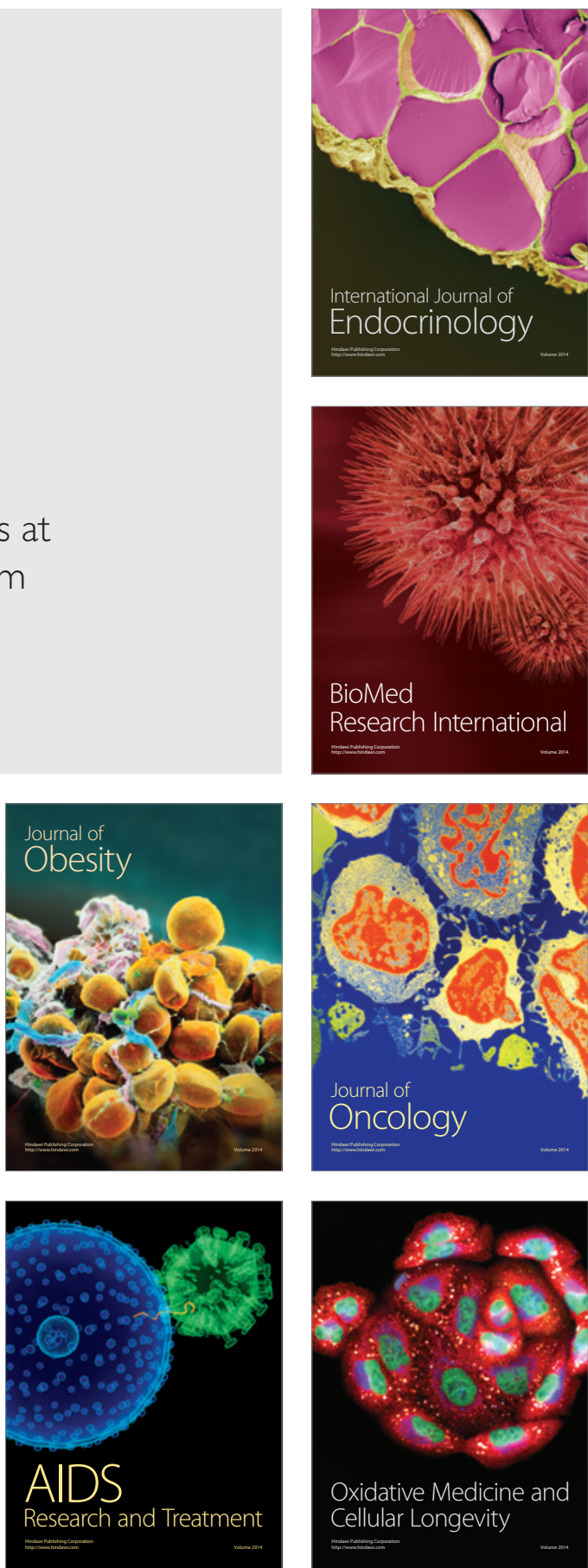